\title{
Does drug treatment reduce the risk of further self-harm or suicide? ${ }^{\dagger}$
} COMMENTARY ON... COCHRANE CORNER

\author{
Katharine Smith \& Mary Jane Attenburrow
}

\begin{abstract}
SUMMARY
Self-harm is a significant social and healthcare problem, with substantial morbidity and healthcare costs. It has strong links to further self-harm and to suicide. The current review is one of three that investigate interventions in preventing recurrence of self-harm, and it focuses on pharmacological treatment. The conclusions are limited by the small number and size of trials identified, and the low quality of evidence. No benefit on recurrence of self-harm was detected in three small trials of antidepressants, but the types studied are ones that are now less commonly used. A small trial of flupentixol suggested a possible benefit on repetition, but this has not been replicated. One small trial of lithium showed no benefit, but this was in contrast to a recent large meta-analysis showing a significant anti-suicidal effect of lithium when used to treat mood disorder. The review highlights important areas for further research.
\end{abstract}

\section{DECLARATION OF INTEREST}

None

\section{Clinical setting}

Self-harm refers to an intentional act of selfpoisoning or self-injury, irrespective of the motivation or apparent purpose of the act (National Collaborating Centre for Mental Health 2004). It includes acts intended to result in death ('attempted suicide'), those without suicidal intent (e.g. to communicate distress, to reduce unpleasant feelings) and those with mixed motivation.

Self-harm is a significant social and healthcare problem. It is often associated with substantial morbidity and healthcare costs, and has strong links to suicide (Sinclair 2011). Between 15 and 25\% of individuals who present to hospital with selfharm repeat this within a year, and 1 to $3 \%$ die by suicide in the same period (Carroll 2014). Of those who die by suicide, over $50 \%$ will have a history of self-harm and at least 15\% will have presented to hospital with self-harm in the preceding year (Gairin 2003). A history of self-harm is the strongest risk factor for suicide across a range of psychiatric disorders, and repetition of self-harm further increases this risk (Zahl 2004).

It is estimated that there are 200000 presentations related to self-harm to general hospitals each year in the UK (Hawton 2007). However, this probably underestimates the true rate, as selfharm often occurs without seeking formal help. Self-harm (unlike suicide) occurs more commonly in females than males and predominantly in those under 35 (Townsend 2014). Although less common in older people, when self-harm occurs in this group it tends to be associated with high suicidal intent and a greater risk of subsequent suicide (Murphy 2012). Self-harm is often associated with acute life events against a background of longer-term difficulties (such as social isolation, relationship or financial difficulties). In addition there is a high prevalence of psychiatric comorbidity (Hawton 2013), including major depression, bipolar disorder, anxiety and substance misuse, often in combination with personality disorders. Given this high prevalence, interventions (pharmacological or psychological) aimed at treating associated psychiatric comorbidity might be anticipated to be effective in reducing future self-harm.

The review in this month's Cochrane corner (Hawton 2015; summary on p. 2, this issue) is an update of an original single review of all treatments for self-harm aimed at reducing repetition (Hawton 1999). The authors have divided the evidence into three updated reviews to allow space for assessment of secondary outcomes, which were not considered in the original review. The present review focuses on pharmacological interventions for adults (the second will consider psychosocial interventions for adults and the third interventions for children and adolescents).

\section{Current pharmacological treatments}

Given the high prevalence of depressive illness in people who self-harm (Hawton 2013), antidepressants (particularly those with lower risk in overdose, such as selective serotonin reuptake
ROUND THE CORNER
Katharine Smith is an honorary consultant psychiatrist at the National Institute for Health Research (NIHR) Oxford cognitive health Clinical Research Facility and Oxford University Department of Psychiatry. Mary Jane

Attenburrow is Clinical Lead at the NIHR Oxford cognitive health Clinical Research Facility, a Senior Research Fellow at Oxford University Department of Psychiatry and an honorary consultant psychiatrist. Correspondence Dr Katharine Smith, NIHR Oxford cognitive health Clinical Research Facility, Warneford Hospital, Oxford OX3 7JX, UK. Email: katharine.smith@psych.ox.ac.uk

${ }^{\dagger}$ See p. 2, this issue 
inhibitors (SSRIs)), are often used in treatment aimed to prevent recurrence of self-harm (Hawton 2010). Mood stabilisers are also used, particularly as there is evidence that when lithium is used to treat people with affective disorders it has specific anti-suicidal effects, including reducing the risk of suicide (Cipriani 2013).

In people with a history of repeated self-harm, especially those with a diagnosis of borderline personality disorder, treatment with antipsychotics may be considered, although there is little evidence for their efficacy in reducing suicidal behaviour (Stoffers 2010). There is also interest in the use of natural products, such as dietary supplementation with omega-3 fatty acids, in a variety of mental disorders, including suicidal behaviour, but there is little convincing evidence of their efficacy at present (Ross 2007).

\section{Methods}

In this 2015 review, Hawton et al searched (in September 2014) for all randomised controlled trials (RCTs) of pharmacological agents or natural products $v$. placebo or any other pharmacological agent for the specific treatment of repeated selfharm in adults. Self-harm had to have occurred in the 6 months prior to trial entry and to have resulted in presentation to clinical services. There were no restrictions on the frequency of the self-harm, or the type of motivation or degree of perceived suicidal intent. However, repetitive self-harm (e.g. headbanging) in the context of intellectual disability was excluded.

The quality of the studies was assessed according to the GRADE criteria (Higgins 2011) (Box 1). The primary outcome measure of the review was the recurrence of self-harm over a maximum followup period of 2 years. Secondary outcomes were treatment adherence, depression, hopelessness, suicidal ideation, suicide and problem-solving.

\section{Results of the review}

The initial review (Hawton 1999) found seven trials of pharmacological treatment, and this 2015 update did not find any new trials or additional information from existing trials. Two ongoing trials (of lithium: Liang 2014; and oral ketamine: Tel-Aviv Sourasky Medical Center 2014) were identified.

All seven trials were RCTs, with a total of 546 participants. The average age was 35.3 years and $63.5 \%$ of participants were female. The risk of bias was unclear in some trials (insufficient reporting of procedures such as masking (blinding) of personnel and outcome assessors) and high in others because of incomplete outcome data and
BOX 1 GRADE quality assessment

The Grading of Recommendations Assessment, Development and Evaluation (GRADE) approach (www.gradeworkinggroup.org), used by the Cochrane collaboration, NICE and publications such as the BMJ, assesses the quality of evidence according to the type and quality of the included trials. 'Low quality' indicates that further research is very likely to have an important impact on the confidence in the estimate of effect and is likely to change the estimate. 'Very low quality' indicates that reviewers are very uncertain about the estimate (Higgins 2011: section 12.2.1).

selective reporting. Overall, the quality of the evidence as assessed by the GRADE criteria was low or very low.

There was no significant effect on the primary outcome, repetition of self-harm, for newergeneration antidepressants (three trials, each of mianserin, nomifensine and paroxetine, analysed together: odds ratio (OR) $0.76,95 \%$ confidence interval (CI) $0.42-1.36, n=243$ ), for low-dose fluphenazine $12 \mathrm{mg} \mathrm{v}$. ultra-low-dose fluphenazine $1.5 \mathrm{mg}(\mathrm{OR}=1.51,95 \% \mathrm{CI} 0.50-4.58, n=53)$, for lithium $(\mathrm{OR}=0.99,95 \%$ CI $0.33-2.95, n=167)$ or for natural products (omega-3 essential fatty acid $(n-3 \mathrm{EFA}):$ OR $=1.33,95 \%$ CI $0.38-4.62, n=49)$. A significant reduction in self-harm repetition was found in a single small trial of flupentixol (flupenthixol) injections $v$. placebo $(\mathrm{OR}=0.09$, $95 \%$ CI $0.02-0.50, n=30$ ), although the overall quality of evidence was very low. We describe ORs and CIs in Boxes 2 and 3.

Information on primary psychiatric diagnoses was reported in only five out of seven trials and, where reported, the most common diagnoses were borderline personality disorder and other personality disorders. Additional comorbid diagnoses were reported in detail in only one trial (Lauterbach 2008). Consequently, it was not possible to make any association between the effects of different types of pharmacological treatment on repetition of self-harm according to diagnostic group.

Secondary outcomes (including suicide) were difficult to assess because of the paucity of included trials, small numbers and incomplete reporting. Therefore no clear conclusions could be drawn on the effect of pharmacological treatments on suicide from this analysis.

\section{Discussion}

The conclusions of this Cochrane review are limited by the small number of trials and trial sizes too small to detect significant differences in 
proportions of patients who repeated self-harm or died by suicide. In addition, the quality of evidence was assessed as low to very low according to the GRADE criteria, suggesting that further research is likely to have an important impact on confidence in the estimate of treatment effectiveness.

Three trials focused on newer-generation antidepressants and showed no effect. However, these trials included drugs that are either no longer used or are in declining use (mianserin, nomifensine). The antidepressants now most commonly used for treatment of depression are SSRIs, but only one drug from this group (paroxetine) was assessed.

Depot flupentixol appeared to reduce repetition of self-harm in one small trial (Montgomery 1979). However, this has not been replicated and similar trials have not yet been conducted with newer oral antipsychotics.

The analysis used in this review found no beneficial effect of lithium for repetition of selfharm, depression, hopelessness, suicidal ideation or suicide in the one small study of individuals with depression who had attempted suicide (Lauterbach 2008). However, a recent systematic review and meta-analysis (Cipriani 2013) that included 48 trials and a total of 6674 participants assessed whether lithium had a specific preventive effect for suicide and self-harm in people with bipolar and unipolar mood disorders. The review found that, although no clear benefits were observed for lithium compared with placebo in preventing self-harm $(\mathrm{OR}=0.60,95 \%$ CI 0.27-1.32), lithium was more effective than placebo in reducing the number of suicides (OR $=0.13,95 \%$ CI $0.03-0.66)$ and deaths from any cause $(\mathrm{OR}=0.38,95 \% \mathrm{CI}$ 0.15-0.95).

\section{Previous guidelines and reviews}

Suicide prevention strategies (such as that from the World Health Organization (2014) and The National Suicide Prevention Strategy for England (Department of Health 2012)) have focused on improving the care of patients presenting with selfharm because of their greatly increased suicide risk and high levels of psychopathology. The National Institute for Health and Care Excellence (NICE) has also produced guidelines on short-term (National Collaborating Centre for Mental Health 2004) and long-term management (NICE 2011).

There are other relevant reviews in this area. A review of pharmacotherapy in 'self-mutilation' suggested benefits for mood stabilisers and antipsychotics, but the effects were less strong when only the data from RCTs were analysed (Smith 2005). A Cochrane review concluded that antipsychotics and mood stabilisers may be

\section{BOX 2 Odds, risk and their ratios}

- The odds is the ratio of the probability that a particular event will occur to the probability that it will not occur; it is usually expressed as a ratio (for example 1:100) or as a decimal (0.01)

- In healthcare the odds is the ratio of the number of people with the event to the number without (e.g. $E_{1} / N_{1}$ or $E_{C} / N_{C}$ in the example below)

- The 'odds' differ from 'risk', which is the probability that an event will occur in relation to the total number of people exposed to that risk (e.g. $E_{1} / T_{1}$ or $E_{C} / T_{C}$ )

- Measures of relative effect express the outcome in one group relative to that in the other; the risk ratio (or relative risk, RR) is the ratio of the risk of an event in the two groups, whereas the odds ratio (OR) is the ratio of the odds of an event

- The OR and RR are similar measures in that they both assess the relative effect of an intervention; both can be used to measure effect in studies with dichotomous outcomes

-When an event is rare, the difference between the OR and the RR is small, but it is greater when events are more common

- If the OR or RR equal 1, this indicates that the estimated effects are the same for both interventions

For example, the results of a clinical trial can be displayed as a $2 \times 2$ table:

\begin{tabular}{|lccc|}
\hline & $\begin{array}{c}\text { Event (e.g. further } \\
\text { self-harm) }\end{array}$ & $\begin{array}{c}\text { No event (e.g. no } \\
\text { further self-harm) }\end{array}$ & Total \\
\hline $\begin{array}{l}\text { Intervention } \\
\text { (e.g. antidepressant) }\end{array}$ & $\mathrm{E}_{\mathrm{I}}$ & $\mathrm{N}_{\mathrm{I}}$ & $\mathrm{T}_{\mathrm{I}}$ \\
\hline $\begin{array}{l}\text { Control } \\
\text { (e.g. placebo) }\end{array}$ & $\mathrm{E}_{\mathrm{C}}$ & $\mathrm{N}_{\mathrm{C}}$ & $\mathrm{T}_{\mathrm{C}}$ \\
\hline
\end{tabular}

$$
\begin{aligned}
R R(\text { relative risk }) & =\frac{\text { Risk of event in intervention group }}{\text { Risk of event in control group }}=\frac{E_{1} / T_{1}}{E_{C} / T_{C}} \\
\text { OR (odds ratio) } & =\frac{\text { Odds of event in intervention group }}{\text { Odds of event in control group }}=\frac{E_{1} / N_{1}}{E_{C} / N_{C}}
\end{aligned}
$$

\section{B0X 3 Confidence intervals}

Results for both individual studies and metaanalyses report a point estimate together with an associated confidence interval (CI).

For example, in the Cochrane review discussed in this article, the meta-analysis of the effects of antidepressants on repetition of self-harm is reported as 'the odds ratio (OR) was 0.76 with a $95 \%$ confidence interval (CI) of 0.42 to $1.36^{\prime}$

The point estimate (0.76) is the best guess of the magnitude and direction of the experimental intervention's effect compared with the control intervention. At first glance, this value of 0.76 might suggest that there is an effect. However, the $\mathrm{Cl}$ describes the uncertainty in this estimate, giving the range of values within which we can be reasonably sure that the true effect actually lies.
A 95\% $\mathrm{Cl}$ means that if someone were to keep repeating the study in other samples from the same population, $95 \%$ of the times the best guess (i.e. the true value of the unknown quantity) will be within the limits of the confidence intervals.

Alternatives such as $90 \%$ and $99 \% \mathrm{Cls}$ are sometimes used.

If the confidence interval is relatively narrow, the effect size is known precisely. If the interval is wider, as in this case $(0.42$ to 1.36) the uncertainty is much greater.

Note that the $\mathrm{Cl}$ in this case encompasses a range of values including an $O R$ of 1 . This means that there is the possibility that the estimated effects are the same for both interventions. Thus, we conclude that there was no significant effect of antidepressants on repetition of self-harm. 
beneficial for symptom reduction in borderline personality disorder, but results were inconclusive for suicidal behaviour (Stoffers 2010). Another Cochrane review found weak evidence for the opioid receptor antagonist naltrexone and for clomipramine in reducing self-injurious behaviour in adults with intellectual disabilities (Rana 2013).

\section{Implications}

Although this 2015 review did not show clear benefits for drug treatment, further research is still needed. The high prevalence of depression among people who self-harm (Hawton 2013) and the strong association between both depression and self-harm and suicide suggests that further trials of antidepressants, particularly of more modern and less toxic ones, are needed. The apparent benefit of flupentixol suggests that there might be value in trials on the use of antipsychotic drugs in the subgroup of patients with repeated selfharm. The beneficial effect of lithium on suicidal behaviours in people with affective disorders shown in the recent meta-analysis (Cipriani 2013) suggests that there should be further assessment of lithium in reducing self-harm in general. In addition to prospective trials, population-wide data on psychiatric diagnosis, pharmacological treatment, self-harm and suicide would be useful in assessing the relationship between medication use and suicidal behaviour.

It is also important that future trials of interventions for self-harm describe the characteristics of the participants in detail (including psychiatric diagnosis) to provide evidence on prediction of response to medication according to clinical subgroups. Trials should assess the full range of outcome measures, including not only self-harm and suicide, but also adherence to treatment, the acceptability of treatment offered and its therapeutic benefit as assessed by ratings of mood. Side-effects of medication should also be assessed, as well as any use of prescribed medication in overdose. Hawton et al plan a separate Cochrane review on psychosocial interventions. It will be interesting to see what further evidence this brings on treating this group of vulnerable patients.

\section{Acknowledgements}

The authors acknowledge support from the NIHR Oxford cognitive health Clinical Research Facility and thank Andrea Cipriani, Associate Professor at Oxford University Department of Psychiatry, for his comments and suggestions. The views expressed are those of the authors and not necessarily those of the NHS, the NIHR or the Department of Health.

\section{References}

Carroll R, Metcalfe C, Gunnell D (2014) Hospital presenting self-harm and risk of fatal and non-fatal repetition: systematic review and metaanalysis. PLoS One, 9: e89944.

Cipriani A, Hawton K, Stockton S, Geddes JR (2013) Lithium in the prevention of suicide in mood disorders: updated systematic review and meta-analysis. BMJ, 346: $\mathfrak{f 6 4 6}$

Department of Health (2012) Preventing Suicide in England: A CrossGovernment Outcomes Strategy to Save Lives. Department of Health.

Gairin I, House A, Owens D (2003) Attendance at the accident and emergency department in the year before suicide: retrospective study. British Journal of Psychiatry, 183: 28-33.

Hawton K, Townsend E, Arensman E, et al (1999) Psychosocial and pharmacological treatments for deliberate self harm. Cochrane Database of Systematic Reviews, 4: CD001764.

Hawton K, Bergen H, Casey D, et al (2007) Self-harm in England: a tale of three cities. Multicentre study of self-harm. Social Psychiatry and Psychiatric Epidemiology, 42: 513-21.

Hawton K, Bergen H, Simkin S, et al (2010) Toxicity of antidepressants: rates of suicide relative to prescribing and non-fatal overdose. British Journal of Psychiatry, 196: 354-8.

Hawton K, Saunders KEA, Topiwala A, et al (2013) Psychiatric disorders in patients presenting to hospital following self-harm: a systematic review. Journal of Affective Disorders, 151: 821-30.

Hawton K, Witt KG, Taylor Salisbury TL, et al (2015) Pharmacological interventions for self-harm in adults. Cochrane Database of Systematic Reviews, 7: CD011777.

Higgins JPT, Green S (eds) (2011) Cochrane Handbook for Systematic Reviews of Interventions Version 5.1.0 (Updated March 2011). Cochrane Collaboration.

Lauterbach E, Felber W, Müller-Oerlinghausen B, et al (2008) Adjunctive lithium treatment in the prevention of suicidal behaviour in depressive disorders: a randomised, placebo-controlled, 1-year trial. Acta Psychiatrica Scandinavica, 118: 469-79.

Liang M (2014) CSP \#590 - Lithium for Suicidal Behavior in Mood Disorders (Lit). ClinicalTrials.gov identifier: NCT01928446.

Montgomery S, Montgomery D, Jayanthi-Rani S, et al (1979) Maintenance therapy in repeat suicidal behaviour: a placebo controlled trial. In Proceedings of the 10th International Congress for Suicide Prevention and Crisis Intervention, June 17-20, 1979, Ottawa, Canada (eds International Association for Suicide Prevention (Canada), Canadian Mental Health Association): 227-9. Association internationale pour la prévention du suicide (Canada).

Murphy E, Kapur N, Webb R, et al (2012) Risk factors for repetition and suicide following self-harm in older adults: multicentre cohort study. British Journal of Psychiatry 200: 399-404.

National Collaborating Centre for Mental Health (2004) Self-Harm: The Short-Term Physical and Psychological Management and Secondary Prevention of Self-Harm in Primary and Secondary Care (Clinical Guideline 16). NICE.

National Institute for Health and Care Excellence (2011) Self-harm in over 8s: Long-Term Management (Clinical Guideline 133). NICE.

Rana F, Gormez A, Varghese S (2013) Pharmacological interventions for self-injurious behaviour in adults with intellectual disabilities. Cochrane Database of Systematic Reviews, 4: CD009084.

Ross BM, Seguin J, Sieswerda LE (2007) Omega-3 fatty acids as treatments for mental illness. Which disorder and which fatty acid? Lipids in Health and Disease, 6: 21.

Sinclair JM, Gray A, Rivero-Arias 0, et al (2011) Healthcare and social services resource use and costs of self-harm patients. Social Psychiatry and Psychiatric Epidemiology, 46: 263-71.

Smith BD (2005) Self-mutilation and pharmacotherapy. Psychiatry, 2 : 28-37. 
Stoffers J, Völlm BA, Rücker G, et al (2010) Pharmacological interventions for borderline personality disorder. Cochrane Database of Systematic Reviews, 6: CD005653.

Tel-Aviv Sourasky Medical Center (2014) Effect of Oral Ketamine Treatment on Suicidal Ideation and Drug Resistant Major Depression: A Clinical and fMRI Study. ClinicalTrials.gov Identifier: NCT02037503.
Townsend E (2014) Self-harm in young people. Evidence-Based Mental Health, 17: 97-9

World Health Organization (2014) Preventing Suicide: A Global Imperative. WHO

Zahl DL, Hawton K (2004) Repetition of deliberate self-harm and subsequent suicide risk: long-term follow-up study of 11583 patients. British Journal of Psychiatry, 185: 70-5. 